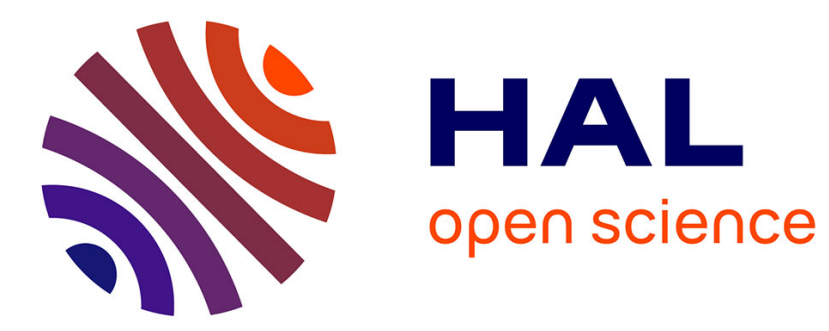

\title{
Les choix des pratiques physiques et sportives des Français : omnivorité, univorité et dissonances Brice Lefevre, Fabien Ohl
}

\section{To cite this version:}

Brice Lefevre, Fabien Ohl. Les choix des pratiques physiques et sportives des Français: omnivorité, univorité et dissonances. Science \& motricité : Revue scientifique de l'Association des Chercheurs en Activités Physiques et Sportives, 2007, 3 (62), pp.81-90. 10.3917/sm.062.0081 . hal-01757052

\section{HAL Id: hal-01757052 https://hal-insep.archives-ouvertes.fr/hal-01757052}

Submitted on 3 Apr 2018

HAL is a multi-disciplinary open access archive for the deposit and dissemination of scientific research documents, whether they are published or not. The documents may come from teaching and research institutions in France or abroad, or from public or private research centers.
L'archive ouverte pluridisciplinaire HAL, est destinée au dépôt et à la diffusion de documents scientifiques de niveau recherche, publiés ou non, émanant des établissements d'enseignement et de recherche français ou étrangers, des laboratoires publics ou privés. 


\title{
LES CHOIX DES PRATIQUES PHYSIQUES ET SPORTIVES DES FRANÇAIS : OMNIVORITÉ, UNIVORITÉ ET DISSONANCES
}

\author{
Brice Lefèvre et Fabien Ohl
}

EDP Sciences | Movement \& Sport Sciences

\author{
$2007 / 3-n^{\circ} 62$ \\ pages 81 à 90
}

ISSN 1378-1863

Article disponible en ligne à l'adresse:

http://www.cairn.info/revue-science-et-motricite-2007-3-page-81.htm

Pour citer cet article :

Lefèvre Brice et Ohl Fabien, « Les choix des pratiques physiques et sportives des Français : omnivorité, univorité et dissonances ",

Movement \& Sport Sciences, $2007 / 3$ n62, p. 81-90. DOI : 10.3917/sm.062.0081

Distribution électronique Cairn.info pour EDP Sciences.

(C) EDP Sciences. Tous droits réservés pour tous pays.

La reproduction ou représentation de cet article, notamment par photocopie, n'est autorisée que dans les limites des conditions générales d'utilisation du site ou, le cas échéant, des conditions générales de la licence souscrite par votre établissement. Toute autre reproduction ou représentation, en tout ou partie, sous quelque forme et de quelque manière que ce soit, est interdite sauf accord préalable et écrit de l'éditeur, en dehors des cas prévus par la législation en vigueur en France. II est précisé que son stockage dans une base de données est également interdit. 


\title{
Les choix des pratiques physiques et sportives des Français : omnivorité, univorité et dissonances
}

\author{
Brice Lefêvere ${ }^{(1)}$ et Fabien Ohl (2)
}

\section{RÉSUMÉ}

La question des usages sociaux de la culture et des liens avec les groupements sociaux continue aujourd'hui à être discutée. Cet article propose d'alimenter les réflexions en interrogeant la cohérence des choix des pratiques physiques et sportives. Ciblant les catégories sociales supérieures, il s'interroge d'un point de vue macrosociologique sur leur éventuelle omnivorité et sur la dissonance de leurs choix de pratiques. A l'aide d'une enquête sur les pratiques sportives des Français $(n=6526)$ il ressort qu'omnivorité et massification des pratiques sont des phénomènes majeurs. Cependant, les catégories supérieures se distinguent par leur " haute omnivorité » et, dans une certaine mesure, leur éclectisme.

Mots-clés : sociologie, sport, goûts, omnivores, dissonance, France.

\section{Choosing a sport: omnivores, univores and dissonance}

\begin{abstract}
The question of the social uses of culture in relation with social groupings continues to be debated. This paper aim is to feed analysis with a study of sport choices coherence. Targeting upper classes and middle upper classes, its questions form a macro-sociological perspective, their omnivority and the dissonance of their sport choices. Through data from an important inquiry $(n=6526)$, we show that omnivority and massification of sport culture are main trends. Nevertheless, upper classes distinguish themselves by a high level of omnivority and, in a way, by their eclectism.
\end{abstract}

Key words: sociology, sport, taste, omnivores, dissonance, France.

(1) Institut National du Sport, 11 avenue du Tremblay 75012 Paris - brice.lefevre@insep.fr.

(2) Faculté des SSP - ISSEP, Université de Lausanne, 1015 Lausanne - Fabien.Ohl@unil.ch 
Dans la sociologie des pratiques sportives française, l'hypothèse d'une cohérence stylistique dans le choix des pratiques culturelles a guidé de nombreux travaux. Les pratiques physiques et sportives ont été abordées en tant qu'élément du style de vie (Bourdieu, 1979) et les recherches ont notamment permis d'observer le rôle de ces pratiques dans l'expression des cultures de classe. La question des usages sociaux de la culture et des liens avec les groupements sociaux a continué à faire débat au sein de la sociologie de la culture (e.g., Lahire, 2004). Cet article, issu d'un travail en cours, propose de prolonger et d'alimenter ces réflexions en interrogeant la cohérence des choix des pratiques sportives.

\section{La question de la signification du choix d'une pratique sportive}

L'analyse des choix fournit des indications sur le type, le taux et l'évolution des pratiques sportives. Elle permet un premier niveau d'observation, facilite les descriptions et donne une vue d'ensemble de la distribution des goûts. Certes, ce n'est pas un indicateur irréprochable, notamment en raison d'une diversité d'usages des pratiques déclarées. Mais les indications sur les modalités ou la fréquence de pratique permettent de mieux cerner les usages.

On peut discuter de la force des liens entre choix et goûts, néanmoins les liens, attestés par de nombreuses enquêtes, existent. Bien sûr, plus on souhaite étudier le goût avec précision, plus l'observation des usages devient importante et moins les méthodes quantitatives sont pertinentes. Néanmoins, l'utilisation de démarches quantitatives ne doit pas être écartée dans la mesure où elles sont incontournables pour décrire et comparer les distributions des choix de pratiques entre différents groupes sociaux. Elles se justifient également pour leur possibilité de quantifier les différences d'usage des pratiques ; le problème des catégories sportives de référence utilisées - l'identification des pratiques à partir du nom de la pratique n'est pas toujours pertinente - pouvant partiellement être neutralisé par la précision, la complémentarité et la diversité des questions posées.

\section{Méthodologie}

Nous utilisons l'enquête nationale sur les « Pratiques sportives des Français en 2000 » de l'INSEP et du Ministère des Sports (Mignon, Truchot, 2002). Elle possède des caractéristiques qui permettent de prendre en compte plusieurs dimensions relatives aux choix (indications sur le type de pratique, le lieu, la quantité, etc.). C'est une enquête qui concerne les pratiques des Français âgés de 15 à 75 ans, réalisée en 2000 à partir d'un échantillon représentatif de 6526 personnes. Elle donne de bonnes indications sur les jugements et les pratiques effectives si l'on utilise une définition très ouverte de la pratique physique ou sportive (au moins une fois dans l'année et relance) et si l'on prend en compte les déclara- 
tions brutes. Les choix semblent être en correspondance avec des goûts, 90 \% des pratiques déclarées dans l'enquête (sur plus de 17 000) sont associées à un jugement positif (« ce qui me plaît »).

Notre analyse de la cohérence des choix s'intéresse d'abord aux catégories supérieures. Comme elles ne représentent que $12 \%$ des effectifs de pratiquants déclarés, il est difficile de vouloir associer un sport aux catégories supérieures si le critère est que les PCS+ doivent représenter la majorité des effectifs. Pour traiter les données nous avons donc choisi d'être attentif aux « petites » différences. Des méthodes descriptives univariées (tris à plat) et bivariées (tris croisés) sont utilisées. Dans les tris croisés le test du khi-2 par case sert à identifier des relations significatives (rarement dues au hasard) d'attraction ou de répulsion et le PEM (Pourcentage de l'Ecart à l'indépendance Maximal) permet de mesurer l'intensité de ces relations (logiciel Tri-deux).

\section{Les pratiques sportives permettent-elles de différencier la population française?}

L'idée est de vérifier si les pratiques sportives différencient encore les populations. Comme le « sens de la distinction » (Bourdieu, 1979) caractérise avant tout les catégories supérieures ${ }^{(3)}$, nous avons choisi de centrer l'analyse sur cette population en essayant de savoir si elle se différencie des autres catégories en ce qui concerne le choix mais aussi le non-choix de pratiques. Pour voir si des activités sont en attraction ou en répulsion avec les catégories supérieures, celles qui concernent au moins trente individus dans l'échantillon sont conservées, soit 70 sur 279 activités déclarées. Néanmoins ces 70 sports représentent tout de même 89 \% du volume total de pratiques déclarées. Le croisement systématique (tests du khi-2 à $1 \%$ ) entre chaque activité sélectionnée et le niveau social mesuré à travers la PCS permet de relever les attractions (notées APS+) et les répulsions (notées APS-).

Sur les 70 pratiques sélectionnées, seulement 14 (soit $20 \%$ ) sont socialement marquées avec une large prédominance des sur-représentations des catégories supérieures (11) par rapport aux sous-représentations (3 cas seulement). Les PCS+ sont donc plus présentes dans une large gamme d'activités que les PCS-. Les données confirment, à ce niveau d'analyse, que même s'il existe encore des pratiques "distinctives » et de rares pratiques " moyennes ou populaires ", la hiérarchisation sociale des sports est peu marquée. Ce qui domine, ce sont des activités largement diffusées dans les diverses catégories sociales qui les pratiquent, les clivages les plus importants étant liés à l'âge et au sexe ${ }^{(4)}$.

(3) La variable synthétique «Catégories supérieures » est constituée par les patrons de l'industrie et du commerce, les cadres et professions intellectuelles supérieures et les assimilés. Les catégories supérieures sont notées PCS+ et les autres (moyennes et populaires) PCS-.

(4) Le format de l'article ne nous permet pas de traiter directement ces variables mais nous les avons prises en compte lors des comparaisons entre PCS en raisonnant toutes choses étant égales par ailleurs. 


\section{Univores et omnivores}

Étant donné la diversité des formes d'engagement dans le sport et de façon générale dans les pratiques culturelles, il est intéressant de prendre en considération la multi-activité. Plusieurs travaux en sociologie de la culture ont suggéré que les frontières sociales jouaient moins sur les pratiques prises individuellement, c'est ce que nous venons partiellement de confirmer, que sur une opposition entre ceux qui peuvent accéder à plusieurs pratiques culturelles, les omnivores, et les autres, les univores (en particulier Peterson \& Kern, 1996). La croissance de l'omnivorité est associée à une transformation du capital culturel, notamment en raison de l'augmentation de la durée moyenne de scolarisation. Pour Fridman et Olivier $(2004,9)$, « le goût éclectique ou omnivore représenterait une nouvelle forme de la disposition cultivée et de la culture légitime ».

L'argumentation utilisant les métaphores zoologiques de l'univore et de l'omnivore pour rendre compte du déplacement des clivages sociaux a été contestée par B. Lahire $(2004,255)$. De ces critiques, nous reprendrons deux remarques. La première, d'ordre méthodologique, invite à traiter de la diversité des choix à partir d'une analyse statistique des individus et non des groupes sociaux. La seconde nuance l'omnivorité comme changement significatif. Il semblerait pour B. Lahire $(2004,258)$ que c'est davantage la sensibilité à l'hétérogénéité des choix individuels que son existence qui soit nouvelle. Néanmoins, en ce qui concerne le sport, l'influence de l'augmentation considérable de l'offre de pratique ne peut être négligée.

\section{L'opposition " univores " et " omnivores ॥ est-elle pertinente pour comprendre les usages sociaux des pratiques sportives?}

On dispose de peu de données sur l'omnivorité sportive. Néanmoins, les travaux ont montré qu'il s'agissait déjà d'une tendance lourde dans l'enquête de l'INSEP de 1987. La pratique de plusieurs sports était déjà sélective socialement (Augustini et al., 1996). Si l'on reprend les travaux de Peterson (Peterson, 2004) sur la transformation des pratiques culturelles, on pourrait supposer que dans l'omnivorisme sportif se reflète une opposition entre les catégories supérieures (omnivores) et les catégories populaires plus univores. Certes, cette opposition produit une vision un peu schématique de la réalité et il est également indispensable de comprendre la cohérence et l'hétérogénéité des choix pour savoir quelles sont les catégories sociales s'appropriant des pratiques non « habituelles ».

En 2000 en France, $83 \%$ des personnes âgées de 15 à 75 ans déclarent au moins une pratique et $17 \%$ ne déclarent pas de pratique. Il y a une grande diversité de pratiques : 279 types de pratiques différentes sont citées. On remarquera que les déclarations de non-pratique concernent peu les PCS+ (répulsion forte avec un PEM à - $40 \%$ ) avec un taux de $7 \%$ contre $18 \%$ pour les autres caté- 
gories sociales. C'est un premier clivage qui place la pratique sportive comme pratique culturelle différenciatrice.

L'analyse de l'univorité (une pratique déclarée) et de l'omnivorité (plus d'une pratique déclarée) des Français indique une moyenne de 2,96 pratiques. Confirmant d'autres travaux, on constate que l'omnivorité est largement majoritaire (tableau 1), elle touche presque trois quarts des pratiquants déclarés alors que l'univorité en concerne un peu plus d'un quart. La basse omnivorité ( 2 et 3 pratiques) touche deux pratiquants sur cinq et la haute omnivorité (4 pratiques et plus) presque une personne sur trois.

Pour mieux comprendre les choix, il apparaît nécessaire d'observer les différences liées à la monopratique et/ou la basse omnivorité et/ou la haute omnivorité. Les résultats sont nuancés ; on ne peut opposer les univores et les omnivores en les associant très nettement à des catégories sociales. La tendance dominante est à l'omnivorité. L'omnivorité de 2 ou 3 pratiques est relativement massifiée sous l'angle des PCS (tableau 2). En effet, les différences entre le niveau supérieur et le niveau inférieur ne sont pas significatives en ce qui concerne la basse omnivorité. Le tableau 2 nous permet de voir que le niveau supérieur et le niveau inférieur se différencient sur les extrêmes : l'univorité et la haute omnivorité. L'univorité est beaucoup plus moyenne ou populaire, le croisement entre nombre de pratiques et PCS montre une forte désaffection des catégories supérieures pour l'univorité (PEM fort, avec en proportion $16 \%$ du niveau supérieur contre $30 \%$ pour le niveau inférieur). La haute omnivorité est plus bourgeoise puisque quatre pratiques déclarées constituent un seuil à partir duquel les différences s'inversent en faveur des catégories favorisées (la haute omnivorité - 4 pratiques et plus - touche $44 \%$ du niveau supérieur contre $29 \%$ du niveau inférieur avec une affinité d'intensité moyenne - PEM à $16 \%$ ).

\section{La diversité des registres de choix}

Plusieurs travaux ont aussi porté sur la diversité et l'hétérogénéité des répertoires utilisés par les différents groupes sociaux. Inspiré de l'idée d'une diversité de répertoires linguistiques mobilisables, DiMaggio $(1987,445)$ a suggéré que la socialisation plurielle de certaines catégories sociales leur donnait la possibilité de mobiliser une diversité de registres d'activité selon le moment et les contextes (culture-switch). Plus récemment, à partir d'une observation des variations intra-individuelles, les travaux de B. Lahire (2004) ont montré que les frontières entre les cultures étaient perméables et que les choix de pratiques homogènes ne sont pas majoritaires, la norme en matière de registres culturels serait plutôt celle de la dissonance ou de l'éclectisme.

Nous proposons d'analyser cette "cohérence » des choix de pratiques sportives. Etant donné que l'omnivorité concerne davantage les catégories supérieures, nous allons centrer notre analyse sur leurs choix. A partir des sur et sous-représentations identifiées, chaque activité est associée positivement ou négativement aux catégories supérieures : une sur-représentation $=1$, une sousreprésentation $=-1$, une activité neutre $=0$. Le lien entre un sport et les PCS+ est à relativiser car aucune activité n'arrive à atteindre le seuil de $50 \%$ de PCS+. 
TABLEAU I

Nombre de pratiques déclarées

\begin{tabular}{|l|c|}
\hline \multicolumn{1}{|c|}{ Nombre de pratiques } & $\%$ \\
\hline monopratique & 28 \\
\hline 2 pratiques & 24 \\
\hline 3 pratiques & 17 \\
\hline 4 pratiques & 12 \\
\hline 5 pratiques & 8 \\
\hline 6 pratiques et plus & 10 \\
\hline Total & 100 \\
\hline
\end{tabular}

Lecture : $28 \%$ des Français de 15 à 75 ans déclarent une pratique.

TABLEAU 2

Nombre de pratiques déclarées et PCS

\begin{tabular}{|l|c|c|c|c|}
\hline Nombre pratiques & PCS+ & PCS- & $\begin{array}{c}\text { PEM sous l'angle } \\
\text { de « PCS+ » }\end{array}$ & $\begin{array}{c}\text { Test du khi-2 } \\
\text { par case }\end{array}$ \\
\hline univore & 16 & 30 & -38 & $* * *$ \\
\hline 2 pratiques & 24 & 24 & - & $\mathrm{ns}$ \\
\hline 3 pratiques & 17 & 17 & - & $\mathrm{ns}$ \\
\hline 4 pratiques & 17 & 12 & 3 & $* *$ \\
\hline 5 pratiques & 12 & 7 & 6 & $* * *$ \\
\hline 6 pratiques et plus & 15 & 10 & 5 & \\
\hline Total & 100 & 100 & & $*$ \\
\hline
\end{tabular}

Lecture : *** : test à $1 \%$ et ${ }^{* *}:$ test à $5 \%$ et ns : non significatif $16 \%$ des PCS+ déclarent une seule pratique et la répulsion est significative avec un PEM à $-38 \%$.

TABLEAU 3

Nombre d'APS+ et d'APS- (bourgeoises) chez les PCS+ et les PCS-

\begin{tabular}{|l|c|c|c|}
\hline \multicolumn{1}{|c|}{ APS+ } & PCS+ & PCS- & Total \\
\hline $\mathbf{0}$ & $48(-)$ & $74(+)$ & 71 \\
\hline $\mathbf{1}$ & $35(+)$ & $20(-)$ & 21 \\
\hline $\mathbf{2}$ & $13(+)$ & $5(-)$ & 6 \\
\hline $\mathbf{3}$ et plus & $5(+)$ & $1(-)$ & 2 \\
\hline Total & 100 & 100 & 100 \\
\hline
\end{tabular}

\begin{tabular}{|l|c|c|c|}
\hline \multicolumn{1}{|c|}{ APS- } & PCS+ & PCS- & Total \\
\hline $\mathbf{0}$ & $55(+)$ & $41(-)$ & 43 \\
\hline $\mathbf{1}$ & $39(-)$ & $49(+)$ & 48 \\
\hline $\mathbf{2}$ et plus & $7(-)$ & $10(+)$ & 10 \\
\hline Total & 100 & 100 & 100 \\
\hline
\end{tabular}

Lecture : $48 \%$ des PCS+ déclarent 0 APS+. 
Les pratiques en affinité avec le niveau supérieur représentent $14 \%$ du volume de pratique total et celles en répulsion $25 \%$. Par ailleurs, seulement $26 \%$ de pratiquants ne possèdent pas d'activité socialement marquée.

Pour observer en quoi se différencient les PCS+ et les PCS- nous avons retenu 8 critères :

- le nombre d'APS+, d'APS- et d'APS neutres ;

- la présence d'activités opposées (1 APS+ et 1 APS-) qui représente une première forme de dissonance ;

- la nature de la balance qui résulte de l'addition de toutes les valeurs des activités ( +1 = une activité «bourgeoise ", -1 une activité " moyenne ou populaire » ou 0 une activité «massifiée » du point de vue des PCS) ;

- la dissonance de la balance (autre forme de dissonance) : il y a dissonance quand elle est positive pour les PCS- et inversement pour les PCS+ ;

- la présence d'au moins une des deux formes de dissonance.

Dans tous les tableaux qui suivent, la notation (+) signifie qu'il y a une attraction et la notation (-) signifie qu'il y a répulsion.

Dans la population des pratiquants, les APS+ sont assez peu présentes puisque seulement $29 \%$ du total de la population pratique au moins une activité typiquement associée aux PCS+ (tableau 3). Évidemment, ces activités ont tendance à être beaucoup plus présentes chez les PCS+ (52\%), avec $35 \%$ de personnes pratiquant une seule activité contre $20 \%$ parmi les PCS-. Néanmoins, la multiactivité «bourgeoise » n'est pas dominante puisque seulement $18 \%$ des PCS+ pratiquent plusieurs activités de ce type.

Les APS- sont plus présentes puisque $48 \%$ des personnes en pratiquent au moins une et $10 \%$ deux et plus (tableau 3). Bien sûr ces activités sont sousreprésentées chez les PCS+, y compris dans la multiactivité (7\% contre $10 \%)$. Notons qu'elles sont tout de même présentes à $46 \%$ dans les catégories supérieures, principalement pour une activité (39\%). L'éclectisme des catégories supérieures semble plus fort que celui des catégories populaires.

La pratique d'activités neutres (massifiées, non associées spécifiquement à un groupe social) est la plus présente (78 \% de la population totale, $87 \%$ des PCS+, $76 \%$ des PCS- en pratiquent au moins une, tableau 4). La majorité des pratiques sont donc peu différenciatrices et la présence de deux activités opposées est rare $(12 \%$, tableau 5$)$. Ce type de dissonance est certes plus présent dans les PCS+ $(20 \%)$ mais reste minoritaire.

Pour mieux lire les données nous avons construit une « balance » des activités. Elle est composée de façon à identifier les pratiques sportives dominantes ${ }^{(5)}$. Le solde est neutre (0 dans le tableau 6 ) pour $34 \%$ des pratiquants, positif pour seulement $19 \%$ et négatif pour $47 \%$ de l'effectif. Les soldes inférieurs à -1 et supérieurs à +1 sont rares.

(5) Les sports sont codés en +1 (liés aux PCS+), -1 (sports populaires) ou en 0 pour les sports non marqués. La dominance est calculée par la somme des valeurs. Lorsque la balance est positive, cela signifie que la dominance est plutôt de pratiques des PCS+, lorsqu'elle est négative cela signifie que les pratiques dominantes sont populaires. 
TABLEAU 4

Nombre d'activités neutres (massifiées) chez les PCS+ et les PCS-

\begin{tabular}{|l|c|c|c|}
\hline Activités neutres & PCS+ & PCS- & Total \\
\hline $\mathbf{0}$ & $13(-)$ & $24(+)$ & 22 \\
\hline $\mathbf{1}$ & 33 & 33 & 33 \\
\hline $\mathbf{2}$ & 26 & 22 & 22 \\
\hline $\mathbf{3}$ & $18(+)$ & $12(-)$ & 13 \\
\hline $\mathbf{4}$ & 6 & 5 & 5 \\
\hline $\mathbf{5}$ et plus & 5 & 5 & 5 \\
\hline Total & 100 & 100 & 100 \\
\hline
\end{tabular}

Lecture : $13 \%$ des PCS+ déclarent 0 activité neutre.

TABLEAU 5

Dissonance liée à la présence d'au moins deux activités opposées chez les PCS+ et les PCS-

\begin{tabular}{|l|c|c|c|}
\hline Présence d'activités opposées & PCS+ & PCS- & Total \\
\hline non & $80(-)$ & $89(+)$ & 88 \\
\hline oui & $20(+)$ & $11(-)$ & 12 \\
\hline Total & 100 & 100 & 100 \\
\hline
\end{tabular}

Lecture : $80 \%$ des PCS+ ne déclarent pas d'activités opposées.

TABLEAU 6

Nature de la balance chez les PCS+ et les PCS-

\begin{tabular}{|l|c|c|c|}
\hline \multicolumn{1}{|c|}{ Balance } & PCS+ & PCS- & Total \\
\hline $\mathbf{- 2}$ et moins & $4(-)$ & $8(+)$ & 8 \\
\hline $\mathbf{- 1}$ & $24(-)$ & $41(+)$ & 39 \\
\hline $\mathbf{0}$ & 35 & 34 & 34 \\
\hline $\mathbf{1}$ & $25(+)$ & $13(-)$ & 14 \\
\hline $\mathbf{2}$ & $9(+)$ & $4(-)$ & 4 \\
\hline $\mathbf{3}$ et plus & $3(+)$ & $1(-)$ & 1 \\
\hline Total & 100 & 100 & 100 \\
\hline
\end{tabular}

Lecture : $4 \%$ des PCS+ ont une balance à -2 et moins.

TABLEAU 7

Proportion de balances dissonantes chez les PCS+ et les PCS-

\begin{tabular}{|l|c|c|c|}
\hline \multicolumn{1}{|c|}{ Balance } & PCS+ & PCS- & Total \\
\hline sport massifié & 35 & 34 & 34 \\
\hline sport de son groupe & $38(-)$ & $49(+)$ & 48 \\
\hline sport dissonant & $28(+)$ & $17(-)$ & 18 \\
\hline
\end{tabular}

Lecture : $28 \%$ des PCS+ ont une balance dissonante. 
TABLEAU 8

Présence d'une des deux formes de dissonance chez les PCS+ et les PCS-

\begin{tabular}{|l|c|c|c|}
\hline \multicolumn{1}{|c|}{ Dissonance } & PCS+ & PCS- & Total \\
\hline neutre (que des APS neutres) & $23(-)$ & $27(+)$ & 26 \\
\hline non & $32(-)$ & $48(+)$ & 46 \\
\hline oui & $45(+)$ & $26(-)$ & 28 \\
\hline Total & 100 & 100 & 100 \\
\hline
\end{tabular}

Lecture : $45 \%$ des PCS+ présentent une forme de dissonance.

En tendance, les PCS+ sont plus liées à une balance positive (37\% dont $12 \%>1$ ) et la balance négative est sous-représentée (un peu plus d'un quart dont seulement $4 \%<-1$ ). C'est-à-dire qu'ici leur éclectisme de pratique est moins lié à des pratiques moyennes ou populaires. Néanmoins, la neutralité caractérise $1 / 3$ de la population et les balances "moyennes ou populaires » (négatives) et les balances «bourgeoises » sont assez proches parmi les PCS+ ( $37 \%$ pour les positives contre $28 \%$ pour les négatives).

Presque la moitié des pratiquants n'affichent pas de balance dissonante (tableau 7), un peu moins d'un cinquième ont une balance dissonante et $1 / 3$ ont une balance neutre. En tendance, les PCS+ sont plus concernées par cette forme de dissonance ( $28 \%$ ) de la balance, leurs choix sont plus éclectiques. L'absence de dissonance de la balance et la neutralité de la balance sont à environ 1/3.

On considère qu'il y a dissonance, par exemple pour la PCS+, quand il y a la présence d'activités opposées ou quand le score final est négatif. Environ la moitié des pratiquants ( $46 \%$, tableau 8 ) n'ont pas de profil dissonant, environ $1 / 4$ ont un profil neutre et $1 / 4$ un profil dissonant. On constate donc une tendance marquée à l'existence de profils plus dissonants parmi les PCS+ (45\% contre $26 \%$ chez les PCS-) alors que l'absence de dissonance (32\%) et la neutralité ( $23 \%)$ sont sous-représentées. Ces niveaux de dissonance dans les choix des pratiques sportives sont plus faibles que le taux moyen de $59 \%$ observé sur l'ensemble des pratiques culturelles des Français (Lahire, 2004, 192). L'éclectisme marque donc clairement les PCS+ sans pour autant être majoritaire.

La massification des activités et l'omnivorité sont des phénomènes d'ampleur même si les catégories supérieures sont plus concernées par la haute omnivorité et la dissonance. Nos données montrent qu'il demeure des pratiques distinctives et d'autres plus populaires, néanmoins c'est une certaine homogénéité entre les catégories qui se dégage à ce niveau d'observation. La question du niveau d'analyse est récurrente. Les niveaux et les méthodes d'observation différents sont difficiles à articuler. Cette approche des goûts sportifs à partir de méthodes quantitatives n'est pas irréprochable mais demeure une méthode intéressante pour aborder les usages sociaux des pratiques sportives et observer la transformation des goûts. Certes, une enquête quantitative ne permet pas d'appréhender toute la diversité des conduites et il existe des différences qui modifient fortement le sens des pratiques, mais elle aide à observer des évolutions dans le temps à partir de données relativement comparables. 


\section{Bibliographie}

Augustini, M., IRLiNGer, P., \& LOUVEAU, C. (1996). Un aspect négligé par l'étude de l'engagement sportif: la multipratique et ses modalités. Loisir et société, 1 , 237-259.

BourdieU, P. (1979). La Distinction, critique sociale du jugement. Paris: Minuit.

DiMaggio, P. (1987). Classification in Art. American Sociological Review, 52, 440-55.

FRIDMAN, V., \& OLIVIER, M., (2004). Présentation : goûts, pratiques culturelles et inégalités sociales : branchés et exclus. Sociologie et sociétés, 36, 1, 3-11.

LAHIRE, B. (2004). La culture des individus. Paris : La découverte.

MignON, P., \& TRUCHOT, G. (2002). Les pratiques sportives en France. Paris: Éditions de l'INSEP.

PETERSON, R., \& KERN, R. (1996). Changing Highbrow Taste: From Snob to Omnivore. American Sociological Review, 61, 900-7.

PETERSON, R. (2004). Le passage à des goûts omnivores : notions, faits et perspectives. Sociologie et Société, 36, 1, 145-164. 